\title{
RVG29-Functionalized Lipid Nanoparticles for Quercetin Brain Delivery and Alzheimer's Disease
}

\author{
R.G.R. Pinheiro' • A. Granja ' J.A. Loureiro ${ }^{2}$ - M.C. Pereira ${ }^{2}$ - M. Pinheiro' • A.R. Neves ',3 (D) - S. Reis '
}

Received: 16 October 2019 / Accepted: 24 June 2020 / Published online: 13 July 2020

(C) Springer Science+Business Media, LLC, part of Springer Nature 2020

\begin{abstract}
Purpose Lipid nanoparticles (SLN and NLC) were functionalized with the RVG29 peptide in order to target the brain and increase the neuronal uptake through the nicotinic acetylcholine receptors. These nanosystems were loaded with quercetin to take advantage of its neuroprotective properties mainly for Alzheimer's disease.
\end{abstract}

Methods The functionalization of nanoparticles with RVG29 peptide was confirmed by NMR and FTIR. Their morphology was assessed by transmission electron microscopy and nanoparticles size, polydispersity and zeta potential were determined by dynamic light scattering. The in vitro validation tests were conducted in hCMEC/D3 cells, a human bloodbrain barrier model and thioflavin $\mathrm{T}$ binding assay was conducted to assess the process of amyloid-beta peptide fibrillation typical of Alzheimer's disease.

Results RVG29-nanoparticles displayed spherical morphology and size below $250 \mathrm{~nm}$, which is compatible with brain applications. Zeta potential values were between -20 and

\section{Highlights}

- Quercetin-loaded lipid nanoparticles functionalized with RVG29 were developed;

- No cytotoxicity of nanoparticles was detected in hCMEC/D3 cell line;

- RVG29-nanoparticles clearly increased in 1.5-fold the permeability across the BBB;

- The nanoparticles confer protection against amyloid-beta fibrillation;

- Great potential for neuroprotection in Alzheimer's disease.

\section{A.R. Neves \\ ana.neves@staff.uma.pt}

LAQV, REQUIMTE, Departamento de Ciências Químicas, Faculdade de Farmácia, Universidade do Porto, 4050-3 I 3 Porto, Portugal

2 LEPABE, Departamento de Ciências da Engenharia, Faculdade de Engenharia, Universidade do Porto, 4500-465 Porto, Portugal

3 CQM - Centro de Química da Madeira, Universidade da Madeira, Campus da Penteada, 9020- I05 Funchal, Portugal
$-25 \mathrm{mV}$. Quercetin entrapment efficiency was generally higher than $80 \%$ and NLC nanoparticles were able to encapsulate up to $90 \%$. The $\mathrm{LDH}$ assay showed that there is no cytotoxicity in hCMEG/D3 cell line and RVG29nanoparticles clearly increased in 1.5-fold the permeability across the in vitro model of blood-brain barrier after $4 \mathrm{~h}$ of incubation compared with non-functionalized nanoparticles. Finally, this nanosystem was capable of inhibiting amyloidbeta aggregation in thioflavin $\mathrm{T}$ binding assay, suggesting its great potential for neuroprotection.

Conclusions RVG29-nanoparticles that simultaneously target the blood-brain barrier and induce neurons protection against amyloid-beta fibrillation proved to be an efficient way of quercetin delivery and a promising strategy for future approaches in Alzheimer's disease.

KEY WORDS amyloid-beta peptide - blood-brain barrier . nanostructured lipid carriers (NLC) · nicotinic acetylcholine receptors · solid lipid nanoparticles (SLN)

\section{INTRODUCTION}

Quercetin is a flavonoid present in many vegetables and fruits that has many beneficial effects in the brain. This compound can protect against oxidative stress, increasing the survival of neuronal cells (1-3). Furthermore, quercetin can induce neuronal differentiation, contributing to maintain cells' balance (4). These properties can be derived from the combined anti-inflammatory and antioxidant properties of this natural compound (5-8). Altogether, these properties can be used to attenuate the adverse environment verified in neurodegenerative diseases and, particularly, help to decrease the protein oxidation, lipid peroxidation and apoptosis of neuronal cells in Alzheimer's disease (9). In fact, some studies show that quercetin is able to attenuate amyloid-beta peptide aggregation $(10,11)$. 
Despite all these promising properties, quercetin has poor solubility in water and undergoes rapid degradation in the body, which difficult its usage in therapy $(12,13)$. In this context, it is necessary to find out solutions to deliver quercetin more efficiently into the places where the compound could be beneficial. Nanotechnology may be an excellent tool to achieve this goal, since nanocarriers can be loaded with quercetin, increase the time of circulation, cross biological barriers, and avoid the immune system and renal clearance, thereby increasing the bioavailability of this compound. Moreover, nanoparticles can be functionalized with specific ligands in order to target specific cells or answer to stimulus in the target site, making them unique carriers for brain delivery $(14,15)$. Ligands that bind to the nicotinic acetylcholine receptors (nAChR) seem to have this capacity, once these receptors are expressed in neuron cells in both pre- and post-synaptic sites and in the brain endothelial cells present in the bloodbrain barrier (BBB) (16-18). The RVG29 peptide is a fragment of 29 aminoacids from the rabies virus glycoprotein, which can interact with these receptors (19-21). Several strategies using RVG29-functionalized nanoparticles have been used for brain delivery (22-24). However, no strategies have been developed using this RVG29 active targeting for quercetin delivery into the brain. Therefore, in this work, we have developed lipid nanoparticles loaded with quercetin and functionalized with RVG29 peptide to take advantage of the neuroprotective properties of quercetin. Indeed, the RVG29nanoparticles here established showed very promising results, improving quercetin permeability through BBB and inhibiting amyloid-beta aggregation, which suggests their great potential for Alzheimer's disease.

\section{MATERIALS AND METHODS}

\section{Materials}

Quercetin (more than 95\% pure) was purchased from SigmaAldrich (St Louis, MO, USA), cetyl palmitate solid lipid was provided by Gattefossé (Nanterre, France), polysorbate 80 (Tween ${ }^{\circledR} 80$ ) was supplied by Merck (Darmstadt, Germany), and miglyol-812 obtained from Acofarma (Madrid, Spain). DSPE-PEG ${ }_{2000}-\mathrm{MAL}$ was purchased from Avanti Polar Lipids (Alabaster, AL, USA) and peptide RVG29 with an additional cysteine on C-terminal (YTIWMPENPRPGTPCDIF TNSRGKRASNG-G) was synthesized by Bachem Group (Germany). EndoGRO-MV Complete Culture Media Kit was purchased from Millipore (Burlington, MA, USA) and supplemented with FBS and Penicillin-Streptomycin obtained from Gibco (Carlsbad, CA, USA) and human bFGF from SigmaAldrich (St Louis, MO, USA). Type I rat collagen was provided by R\&D Systems (Minneapolis, USA), while PBS, trypsin, trypan blue, triton X-100, lucifer yellow and FITC were also obtained from Sigma-Aldrich (St Louis, MO, USA). A $\beta$ (1-42) peptide (purity $>95 \%$, MW 4514.14) was purchased from Selleck Chemicals.

\section{Preparation of Nanoparticles}

Lipid nanoparticles can be subdivided mainly in solid lipid nanoparticles (SLN) and nanostructured lipid carriers (NLC) (25). They were produced by hot homogenization followed by sonication, a technique previously developed in our group (26-28). SLN are composed by a solid lipid (cetyl palmitate) and NLC are composed by the same solid lipid (cetyl palmitate) and also a liquid lipid (miglyol-812). Polysorbate 80 was used as surfactant in both synthesis (Table I). The lipid phase (lipids, surfactant and quercetin or FITG) was previously warmed up at $80^{\circ} \mathrm{C}$ and then mixed to the aqueous buffer (PBS) at the same temperature. The mixture was then subjected to hot homogenization in an Ultra-Turrax T25 Janke and Kunkel IKA-Labortechnik, Staufen, Germany), followed by sonication in a Sonics and Materials Vibra-Cell ${ }^{\mathrm{TM}}$ GV18 (Newtown, CT, USA) to produce nanoparticles. SLN were homogenized for $30 \mathrm{~s}$ (12,000 rpm) and sonicated for $5 \mathrm{~min}$ (80\% intensity), while NLC were stirred for $2 \mathrm{~min}(12,000 \mathrm{rpm})$ and sonicated for $15 \mathrm{~min}(70 \%$ intensity).

Table I Composition of the Synthetized Lipid Nanoparticles (SLN - Solid Lipid Nanoparticles and NLC - Nanostructured Lipid Carriers) with and without Functionalization with RVG29 Peptide

\begin{tabular}{|c|c|c|c|c|c|c|}
\hline Formulation code & Cetyl palmitate (mg) & Miglyol-8I2 (mg) & Polysorbate 80 (mg) & DSPE-PEG-RVG29 (mL) & Quercetin (mg) & $\mathrm{PBS}(\mathrm{mL})$ \\
\hline SLN & 500 & 0 & 150 & 0 & 0 & 4.4 \\
\hline SLN-RVG & 500 & 0 & 150 & I & 0 & 4.4 \\
\hline SLN Quercetin & 500 & 0 & 150 & 0 & 10 & 4.4 \\
\hline SLN-RVG Quercetin & 500 & 0 & 150 & I & 10 & 4.4 \\
\hline NLC & 350 & 150 & 150 & 0 & 0 & 4.4 \\
\hline NLC-RVG & 350 & 150 & 150 & I & 0 & 4.4 \\
\hline NLC Quercetin & 350 & 150 & 150 & 0 & 10 & 4.4 \\
\hline NLC-RVG Quercetin & 350 & 150 & 150 & I & 10 & 4.4 \\
\hline
\end{tabular}




\section{Functionalization of Nanoparticles with RVG29}

For surface modification of nanoparticles with RVG29 peptide, DSPE-PEG-MAL was previously conjugated to RVG29. DSPE-PEG-MAL is a 1,2-distearoyl-sn-glycero-3phosphoethanolamine (DSPE) associated with polyethylene glycol (PEG) and terminal maleimide groups (MAL). The maleimide groups can react with thiol groups, thereby forming thioether bonds (Fig. 1). Hence, RVG29 with cysteine on C-terminal was prepared in PBS (5 mg/mL, pH 7.0) followed by the addition of 1-fold DSPE-PEG-MAL. The mixture was allowed to react at room temperature (20$25^{\circ} \mathrm{C}$ ) for $24 \mathrm{~h}$, thus forming the conjugate DSPE-PEGRVG29. The conjugate was then dialyzed in a $10 \mathrm{kDa}$ MWCO SnakeSkin Dialysis Tubing against $500 \mathrm{~mL}$ PBS at $37^{\circ} \mathrm{C}$, overnight, to remove the excess of RVG29 and byproducts. Finally, nanoparticles were produced as previously described but incorporating $1 \mathrm{~mL}$ of DSPE-PEG-RVG29 conjugate in the lipid phase composition (Table I).

\section{Nuclear Magnetic Resonance Spectroscopy}

NMR spectroscopy was used to confirm the synthesis of DSPEPEG-RVG29 conjugate using a Bruker Avance III 600 HD spectrometer (Bruker, Massachusetts, United States), operating at $600.13 \mathrm{MHz}$ for ${ }^{\mathrm{l}} \mathrm{H}$, with $5 \mathrm{~mm}$ CryoProbe Prodigy, capable of producing $50 \mathrm{G} \mathrm{cm}^{-1}$ magnetic field pulsed gradients in the $\mathrm{z}$ direction. Deuterium oxide $\left(\mathrm{D}_{2} \mathrm{O}\right)$ was used as a solvent and ${ }^{1} \mathrm{H}$ NMR experiments were performed with water suppression using excitation sculpting with gradients, at $300 \mathrm{~K}$ with a spectral width of $10,000 \mathrm{~Hz}$. The chemical shifts of the ${ }^{1} \mathrm{H}$ NMR signals were referred to trimethylsilylpropanoic acid-d4 (TMSP-d4) absorption frequency, as an internal reference.

\section{Fourier Transform Infrared Spectroscopy}

Fourier transform infrared spectroscopy (FTIR) was also used to confirm the functionalization of the nanoparticles with RVG29 ligand. The samples were previously lyophilized at $-85^{\circ} \mathrm{C}$ and 0.76 Torr using a LyoQuest -85 freeze dryer (Telstar, Terrassa, Spain). The infrared spectra of the lyophilized nanoparticles were obtained using a Frontier FTIR Spectrometer from PerkinElmer (Santa Clara, California, USA).

\section{Transmission Electron Microscopy}

Transmission electron microscopy (TEM) was used to analyze nanoparticles morphology. The samples were analyzed using a Jeol JEM 1400 transmission electron microscope (Tokyo) mounted on 300 mesh form var. copper grids. Uranyl acetate was used as contrast agent and images were obtained using a Gatan SC 1000 ORIUS CCD camera (Warrendale, PA, USA).

\section{Dynamic Light Scattering}

The mean hydrodynamic diameter of nanoparticles was measured by dynamic light scattering (DLS) using a particle size analyzer (Brookhaven Instruments, Holtsville, NY, USA). All

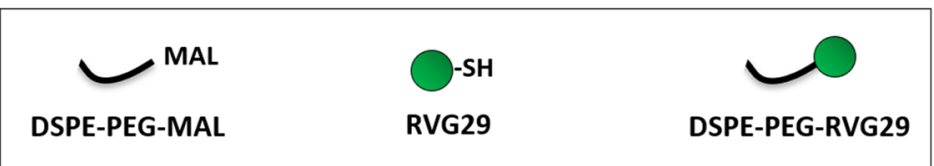

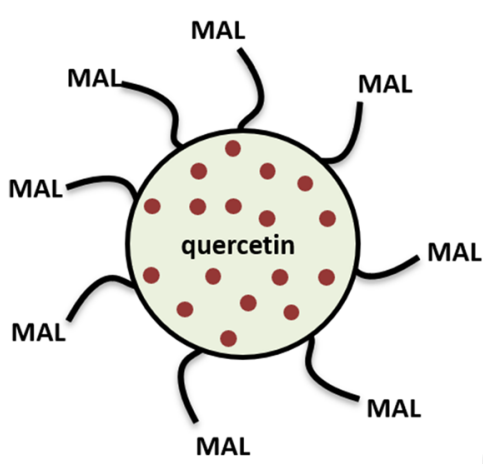

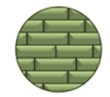

SLN

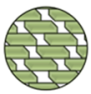

NLC
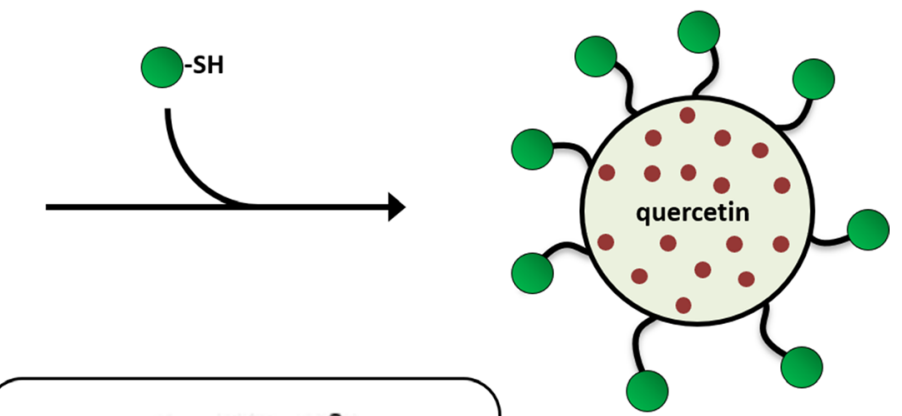

Fig. I Schematic representation of nanoparticles functionalization with RVG29 peptide (not drawn to scale). 
samples were diluted in PBS by a factor of 1:400. All determinations were made at $25^{\circ} \mathrm{C}$ with a light incidence angle of $90^{\circ}$. The hydrodynamic diameter followed a Gaussian distribution and polydispersity index was determined according to the width of particle size distribution.

\section{Zeta Potential Analyzer}

The zeta potential of nanoparticles was calculated by measuring the electrophoretic mobility in a zeta potential analyzer (Brookhaven Instruments, Holtsville, NY, USA). All samples were diluted in PBS by a factor of 1:400 and all the measurements were performed at $25^{\circ} \mathrm{C}$.

\section{Entrapment Efficiency and Loading Capacity Determination}

The entrapment efficiency (EE) was determined by an indirect method, quantifying the amount of quercetin that was not encapsulated and comparing to the total amount of quercetin that was previously added to each formulation. By this way, $2 \mathrm{~mL}$ of 1:200 diluted nanoparticles were filtered through a $3.0 \mu \mathrm{m}$ Millipore-type SSWP membrane filter which retained the unentrapped quercetin, while nanoparticles could cross through the membrane filters. The quercetin retained in the filter was then recovered using $2 \mathrm{~mL}$ of acetonitrile. Finally, a spectrophotometer (V-660 Jasco, Easton, MD, USA) was used to measure the absorbance of quercetin at $367 \mathrm{~nm}$ and quercetin EE was calculated as follows: EE $(\%)=\frac{\text { Total quercetin-Unentrapped quercetin }}{\text { Total quercetin }} \times 100$.

Loading capacity (LC) was obtained by calculating the ratio between the amount of encapsulated quercetin and the total amount of lipids and surfactant present in the nanoparticles, as follows:

$L C(\%)=\frac{\text { Total quercetin }- \text { Unentrapped quercetin }}{\text { Total lipid and surfactant amount }} \times 100$

\section{hCMEC/D3 Cell Culture}

This brain endothelial cell line hCMEC/D3 was obtained from Institut National de la Santé et de la Recherche Médicale (INSERM, Paris, France) and was used as a BBB model system $(29,30)$. Cells were seeded in a concentration of $2.5 \times 10^{4}$ cells $/ \mathrm{cm}^{2}$ in flasks pre-coated with $0.1 \mathrm{mg} / \mathrm{mL}$ type $\mathrm{I}$ rat collagen and grown at $37^{\circ} \mathrm{C}, 5 \%$ $\mathrm{CO}_{2}$ in EndoGRO medium supplemented with Penicillin-Streptomycin (1\%), Fetal Bovine Serum (5\%), Ascorbic Acid $(50 \mu \mathrm{g} / \mathrm{mL})$, Heparin Sulfate $(0.75 \mathrm{U} / \mathrm{mL})$, Hydrocortisone Hemisuccinate $(1.0 \mu \mathrm{g} / \mathrm{mL})$, L-Glutamine $(10 \mathrm{mM})$ and Epidermal Growth Factor $(5 \mathrm{ng} / \mathrm{mL})$.

\section{LDH Cytotoxicity Assay}

Lactate dehydrogenase $(\mathrm{LDH})$ assays were conducted in order to evaluate cytotoxicity and nanoparticles potential for damaging cell membranes. $\mathrm{LDH}$ is an enzyme that is released from cells to the surrounding cell culture supernatant when cell membranes are damaged or disrupted. Cells were seeded in 96-well plates $\left(10^{4}\right.$ cells per well) precoated with type I rat collagen. After $20 \mathrm{~h}$ of incubation at $37^{\circ} \mathrm{C}$ and $5 \% \mathrm{CO}_{2}$, different concentrations of quercetin-loaded nanoparticles (functionalized and nonfunctionalized) were incubated with the cells for $4 \mathrm{~h}$. EndoGRO medium and TX-100 sample were also used as negative and positive controls for cytotoxicity, respectively. After the incubation time, the medium of each well was collected and centrifuged (250 g for $10 \mathrm{~min}, \mathrm{RT}$ ) and the supernatant separated for further LDH quantification assay (LDH detection kit, Takara Bio Inc., Shiga, Japan). After treatment with catalyst and dye solutions for $20 \mathrm{~min}$ at RT in the dark, absorbance was read at 490 and 690 nm using a Synergy ${ }^{\text {TM }}$ HT Multi-mode Microplate Reader (BioTek Instruments Inc., Winooski, VT, USA). Cytotoxicity was expressed as a percentage and compared to the maximum cytotoxicity of TX-100 sample.

\section{Transwell Permeability Assay}

Transwell devices with six-well polyester inserts (pore size $0.4 \mu \mathrm{m}$ and cell growth area of $4.67 \mathrm{~cm}^{2}$ ) were pre-coated with type I rat collagen, followed by hCMEC/D3 cells seeding in a density of $2 \times 10^{5}$ cells per insert. After 7 days, the barrier integrity of cell monolayers was checked using a small reference molecule (Lucifer Yellow) with a well reported effective permeability coefficient $\left(\mathrm{P}_{\text {eff }}\right)$ of $1.33 \times$ $10^{-3} \mathrm{~cm} / \mathrm{min}$ (31). The permeability assay was performed at $37^{\circ} \mathrm{C}$ in a $5 \% \mathrm{CO}_{2}$ incubator using functionalized and non-functionalized nanoparticles previously loaded with the fluorescent probe FITC $(0.4 \mathrm{mg} / \mathrm{mL})$. The nanoparticles permeability was calculated according to the amount of fluorescence $(495 / 519 \mathrm{~nm})$ measured in the receptor compartment after $0.5,1,2,3$ and $4 \mathrm{~h}$ of incubation time.

\section{Amyloid-Beta Peptide Preparation}

A $\beta$ (1-42) peptide was previously dissolved in HFIP (1,1,1,3,3,3-hexafluoro-2-propanol) at $1 \mathrm{mg} / \mathrm{mL}$ to solve the residual peptide aggregation, by disrupting intermolecular $\mathrm{H}$ bonds (32-34). This solution was then evaporated with nitrogen flow and under vacuum and the resulting peptide film was dissolved in dimethyl sulfoxide at $9 \mathrm{mg} / \mathrm{mL}$. 


\section{Thioflavin T Binding Assay and Fluorescence Measurements}

Thioflavin T (ThT) binds to amyloid fibril structures emitting a strong fluorescence $(33,35)$. Solutions of $\mathrm{A} \beta(1-42)$ peptide were incubated at $37^{\circ} \mathrm{C}$ in 96 -well plates at $50 \mu \mathrm{M}$ concentration with different conditions: $30 \mu \mathrm{M}$ quercetin, quercetinloaded nanoparticles (functionalized and non-functionalized) and unloaded nanoparticles. The ThT solution in PBS buffer $(0.7 \mathrm{mg} / \mathrm{mL}, \mathrm{pH} 7.4)$ was added to each well and the fluorescence intensity $\left(\lambda_{\text {exc }} / \lambda_{\text {em }}, 450 / 482 \mathrm{~nm}\right)$ was measured (every 30 min, during $24 \mathrm{~h}$ ) in a Biotek Synergy 2 fluorescence spectrometer (Winooski, Vermont, USA) for detection of ThTamyloid fibrils conjugates (36).

\section{Statistical Analysis}

Statistical analysis was performed using SPSS software (v 24.0; IBM, Armonk, NY, USA). The measurements were repeated three times and data were expressed as mean \pm SD. Data were analyzed using one-way analysis of variance (one-way ANOVA), followed by Bonferroni, Tukey and Dunnett posthoc tests. A $p$ value lower than 0.05 was considered statistically significant.

\section{RESULTS AND DISCUSSION}

\section{'H-NMR Characterization of RVG29-Functionalized Conjugate}

Figure 2 shows the ${ }^{1} \mathrm{H}$ NMR spectra resulted from the coupling of RVG29 peptide to DSPE-PEG-MAL. In the spectrum of DSPE-PEG-MAL, one can observe characteristic peaks of DSPE, $\mathrm{PEG}_{2000}$ repetitions and the maleimide group.

The proton peaks at $0.85 \mathrm{ppm}\left(\mathrm{CH}_{3}\right), 1.30 \mathrm{ppm}\left(\mathrm{CH}_{2}\right)$, $1.60 \mathrm{ppm}\left(\mathrm{CH}_{2} \mathrm{CH}_{2} \mathrm{CO}\right), 2.35 \mathrm{ppm}\left(\mathrm{CH}_{2} \mathrm{CO}\right)$ and $4.25 \mathrm{ppm}$ $\left(\mathrm{PO}_{4} \mathrm{CH}_{2} \mathrm{CH}\right)$ are attributed to DSPE phospholipid $(21,37)$. At $3.75 \mathrm{ppm}$ it is possible to visualize the $\mathrm{CH}_{2} \mathrm{CH}_{2} \mathrm{O}$ peak of the $\mathrm{PEG}_{2000}$ repeat units $(21,37)$, while the peak at $6.70 \mathrm{ppm}$ confirms the presence of the maleimide group in DSPE-PEGMAL spectrum $(21,37,38)$. After functionalization with RVG29, the peaks which correspond to DSPE and PEG $_{2000}$ remain in the NMR spectrum of DSPE-PEG-RVG29. However, the maleimide peak at $6.70 \mathrm{ppm}$ completely disappeared, indicating the successful addition between the maleimide group of DSPE-PEG-MAL and the thiol moieties of RVG29 $(21,37,38)$. Besides, it is possible to observe a peak at $2.10 \mathrm{ppm}$ related to the presence of RVG29 amino groups and also the characteristic peaks of aromatic protons of RVG29 amino acids at 6.00 to 7.60 in the ${ }^{1} \mathrm{H}$ NMR spectrum of DSPE-PEG-RVG29 conjugate. Therefore, the NMR spectra proved the successful functionalization of DSPE-PEG 2000 with RVG29 peptide.

\section{FTIR Characterization of RVG29-Functionalized Nanoparticles}

The functionalization of nanoparticles with RVG29 peptide was confirmed using infrared spectroscopy. In Fig. 3, it is possible to visualize bands that correspond to the $\mathrm{N}-\mathrm{H}$ stretching vibrations $\left(3300 \mathrm{~cm}^{-1}\right), \mathrm{N}-\mathrm{H}$ bending vibrations $\left(1550 \mathrm{~cm}^{-1}\right)$ and $\mathrm{C}=\mathrm{O}$ stretching vibrations $\left(1650 \mathrm{~cm}^{-1}\right)$, which are characteristic of peptide bonds between amino acids present in the RVG29 peptide spectra. These bands are also present in the FTIR spectra of RVG29functionalized SLN and NLC, indicating the successful functionalization of nanoparticles and corroborating with the NMR results.

\section{Morphology Determination}

Figure 4 contains the TEM images of both types of nanoparticles (SLN and NLC), showing a size around $200 \mathrm{~nm}$, spherical shape and uniform morphology. Besides, the encapsulation of quercetin and the functionalization with RVG29 peptide did not seem to change the nanoparticles size and morphology. TEM images also revealed no signs of aggregation, indicating that nanoparticles are stable even when functionalized with RVG29 peptide and when quercetin is loaded.

\section{Physicochemical Characterization and Nanoparticles Stability}

\section{Particle Size Measurements}

The hydrodynamic diameter results are depicted in Table II. All nanoparticles showed a size lower than $250 \mathrm{~nm}$, which is suitable for brain applications, facilitating the BBB crossing (39). In Fig. 5a one can see the results over time in order to evaluate the stability of RVG29-functionalized nanoparticles and it is possible to conclude that nanoparticles retain their size below $250 \mathrm{~nm}$ over 3 months, at room temperature.

These results corroborate with TEM analysis where no visible aggregation was registered. However, it is reasonable that the nanoparticles size obtained by DLS is slightly higher than by TEM, since DLS determines the hydrodynamic diameter of nanoparticles, accounting with the hydration sphere composed by water, ions and counter-ions. NLC were found to be slightly lower than SLN $(p<0.05)$, while the functionalization with RVG29 showed to increase the mean average size of both types of lipid nanoparticles $(p<0.05)$, except for quercetin-loaded SLN (Table II). Concerning the polydispersity index, all values seem to be lower than 0.3 , even 
Fig. 2 'H NMR spectra of DSPEPEG-MAL, RVG29 and DSPEPEG-RVG29 samples.

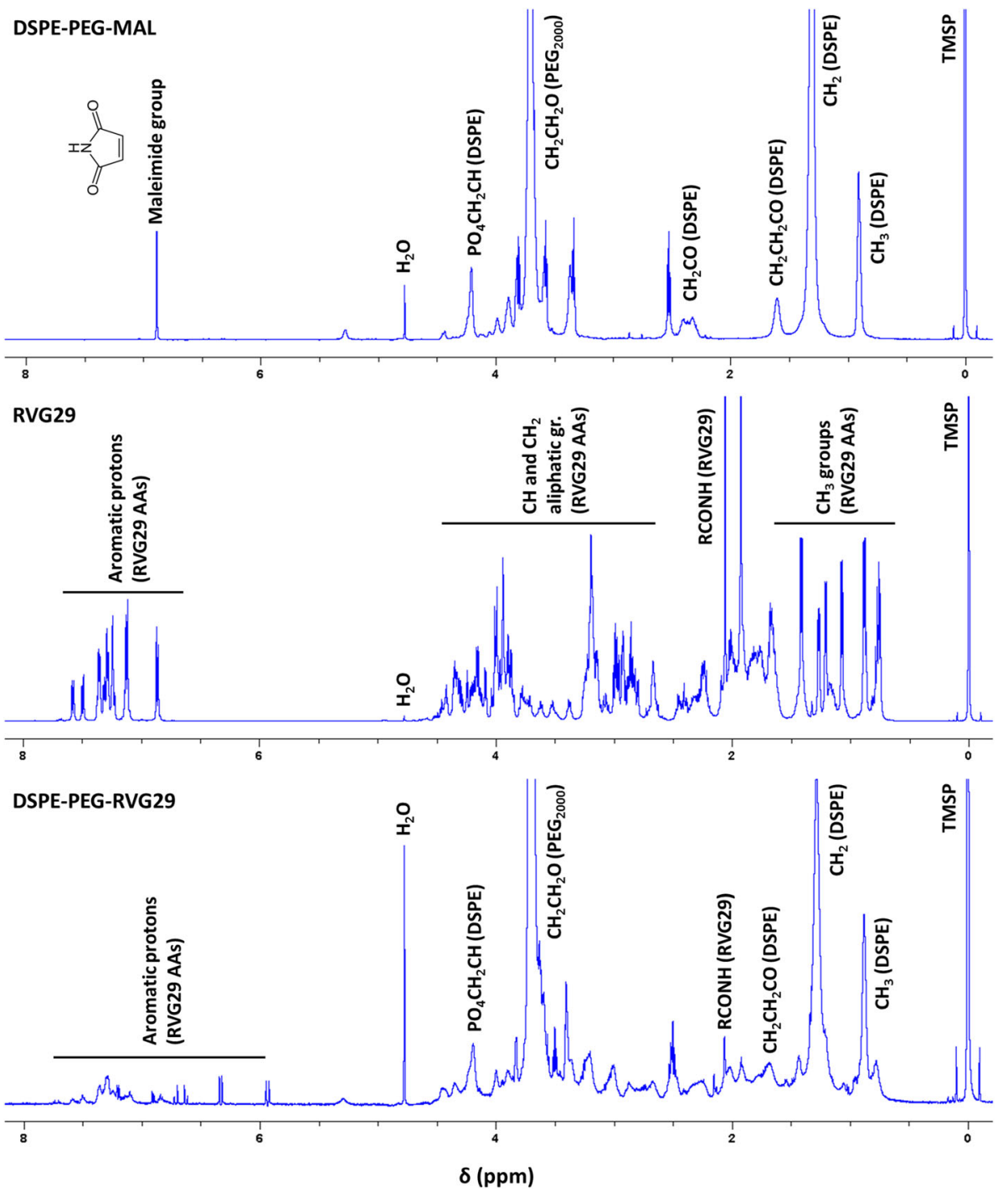

after 3 months of storage (Fig. 5b), suggesting an acceptable size distribution with low variability and no aggregation of particles, typical of the high shear homogenization and ultrasonication methods.

\section{Zeta Potential Measurements}

Zeta potential of RVG29-functionalized nanoparticles varied between $-20 \mathrm{mV}$ and $-30 \mathrm{mV}$ (Table II) and remained almost unchanged after 3 months of study, at room temperature (Fig. 5c), which guarantees the nanoformulations stability. These values were measured in PBS solution rather than water to ensure the stability of the thioether bound between RVG29 peptide and the maleimide group. As a consequence, the ions present in PBS may reduce the nanoparticles superficial charge, thereby decreasing the values of measured zeta potential. Besides that, neither RVG29 functionalization nor quercetin encapsulation seems to significantly interfere with the nanoparticles zeta potential, providing good stability results over time.

\section{Quercetin Entrapment Efficiency and Loading Capacity}

Entrapment efficiency $(\% \mathrm{EE})$ is the percentage of drug that is successfully entrapped into the nanoparticles compared to the total drug added to the formulation. However, it is also very pertinent to calculate the Loading Capacity (\%LC) which is the amount of drug loaded per unit weight of nanoparticles, indicating the percentage of mass of the nanoparticle that is due to the encapsulated drug. Hence, $\% \mathrm{LC}$ was calculated by the amount of total entrapped drug divided by the total nanoparticle weight, considering the amount of lipids and surfactant present in the nanoparticles. The results in Table II revealed that both SLN and NLC were able to encapsulate high amounts of quercetin $\% \mathrm{EE}=80-90$ and $\% \mathrm{LC}=2-3)$, which correspond to 8-9 $\mathrm{mg}$ of quercetin entrapped inside the nanoparticles. Moreover, NLC presents 
Fig. 3 Infrared spectra obtained by FTIR for SLN (A) and NLC (B) before and after functionalization with RVG29 peptide. Note: RVG29 sample was used as a reference to compare with the functionalized nanoparticles.
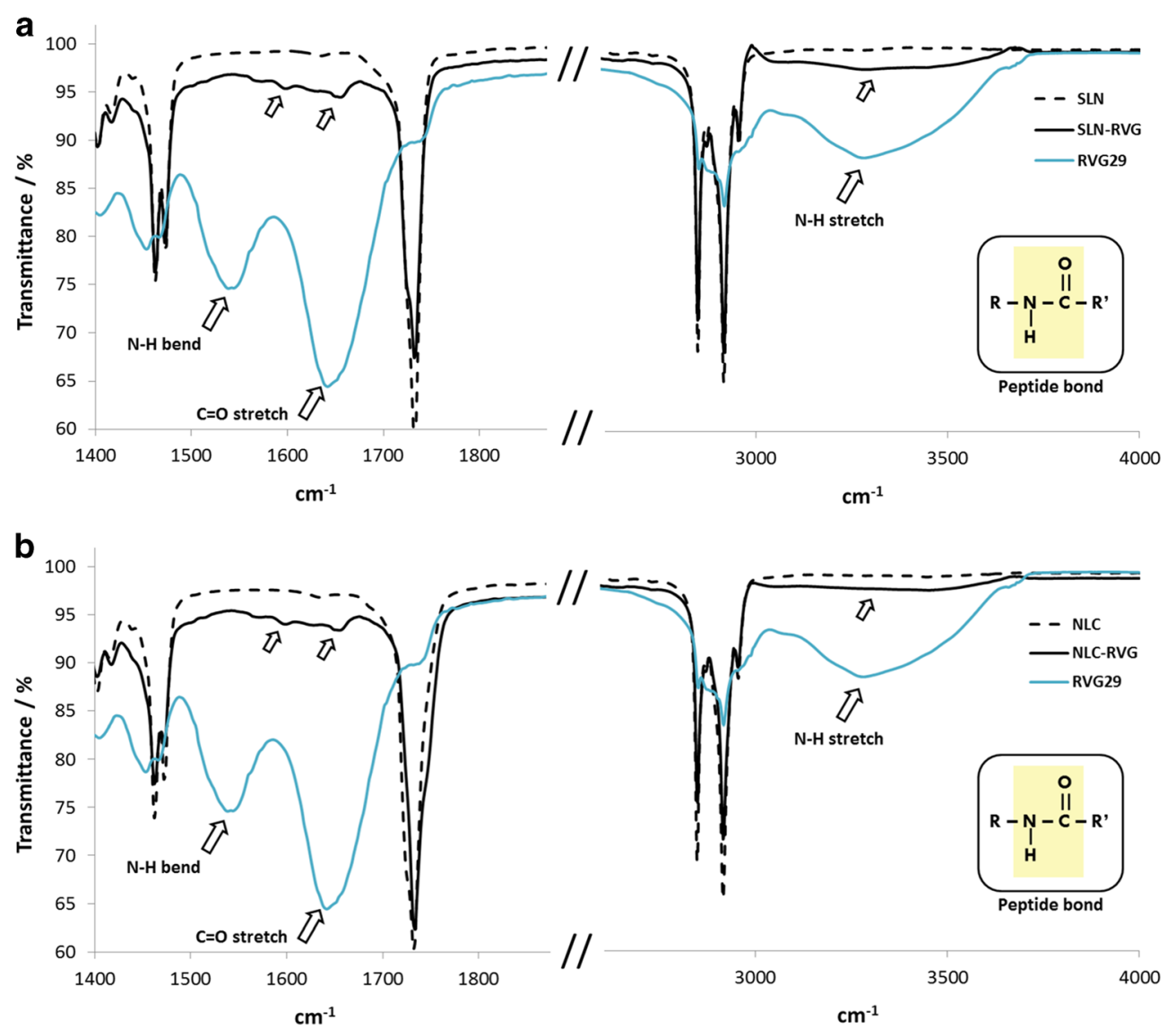

higher values than SLN, probably due to the presence of the liquid lipid that confers a less ordered crystalline structure with more cavities in the lipid matrix, allowing a better accommodation of quercetin $(40,41)$. Furthermore, despite not being statistically significant, a slight tendency for entrapment efficiency to decrease after 3 months for SLN-RVG can be assumed (Fig. 5d). This fact can be justified by the highly ordered crystalline structure of SLN which may trigger a premature release over time. However, even after 3 months at room temperature, SLN-RVG still have a high amount of compound associated with the nanoparticles. On the other hand, this entrapment decrease did not occur for NLC-RVG probably due to the less ordered nanoparticles crystalline structure that avoids the triggered release effect.
SLN

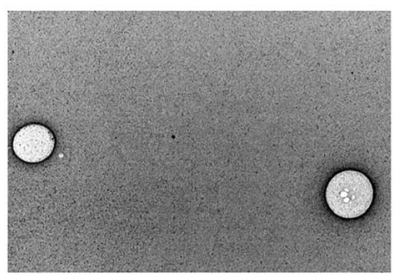

No funct.

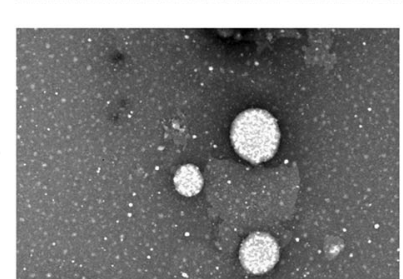

SLN Quercetin
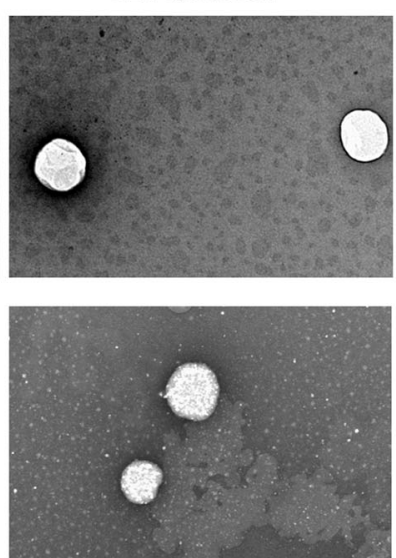

NLC
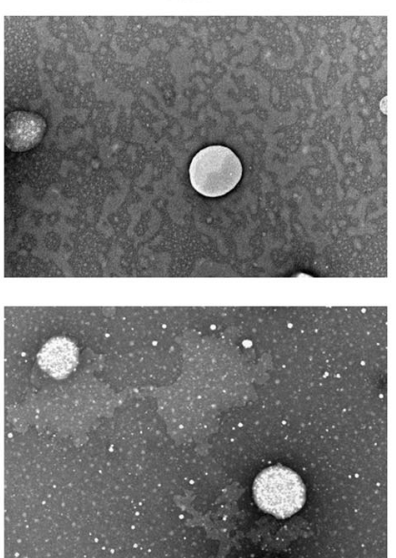

NLC Quercetin
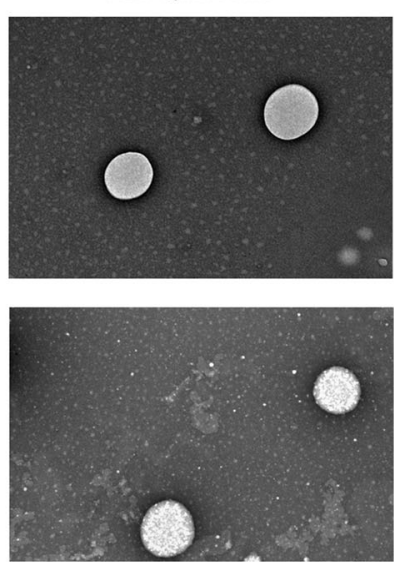

$\overline{200 \mathrm{~nm}}$

Fig. 4 Transmission electron microscopy images of lipid nanoparticles: non-functionalized (upper side) and RVG29-functionalized nanoparticles (lower side). Magnification: $30,000 \times$. 
Table II Characterization of RVG29-Functionalized SLN and NLC Compared to Non-Functionalized Ones, According to their Size, Polydispersity Index, Zeta Potential and Quercetin Entrapment Efficiency

\begin{tabular}{|c|c|c|c|c|c|}
\hline Formulation code & Particle size (nm) & Polydispersity index & Zeta potential (mV) & Entrapment efficiency (\%) & Loading capacity (\%) \\
\hline SLN & $167 \pm 6$ & $0.13 \pm 0.02$ & $-31 \pm 6$ & - & - \\
\hline SLN-RVG & $246 \pm 29 *$ & $0.09 \pm 0.03$ & $-26 \pm 7$ & - & - \\
\hline SLN Quercetin & $195 \pm 4$ & $0.09 \pm 0.02$ & $-3 \mid \pm 5$ & $81 \pm 12$ & $2.7 \pm 0.4$ \\
\hline SLN-RVG Quercetin & $201 \pm 23$ & $0.13 \pm 0.07$ & $-20 \pm 7$ & $84 \pm 10$ & $2.8 \pm 0.3$ \\
\hline NLC & $147 \pm 5$ & $0.05 \pm 0.01$ & $-24 \pm 2$ & - & - \\
\hline NLC-RVG & $224 \pm 5 *$ & $0.07 \pm 0.05$ & $-24 \pm 1$ & - & - \\
\hline NLC Quercetin & $170 \pm 5$ & $0.12 \pm 0.03$ & $-28 \pm 2$ & $97 \pm 10$ & $3.2 \pm 0.3$ \\
\hline NLC-RVG Quercetin & $222 \pm 22 *$ & $0.23 \pm 0.08$ & $-25 \pm 3$ & $93 \pm 2$ & $3.1 \pm 0.1$ \\
\hline
\end{tabular}

Note: All values represent the mean \pm standard deviation $(n=3)$. * denotes statistically significant differences between RVG29-functionalized nanoparticles and non-functionalized ones $(P<0.05)$.

\section{Cytotoxicity Study}

The RVG29-nanoparticles cytotoxicity was evaluated using the lactate dehydrogenase (LDH) assay in hCMEC/D3 cells line in order to verify if lipid nanoparticles are safe for brain endothelial cells. In this assay, the LDH, which is released when the cell membrane is damaged, is quantified by the formation of a red formazan product resulting from redox reactions $(42,43)$. The extension of this reaction can be monitored by measuring absorbance, which is proportional to cell death levels. The results in Fig. 6a show that cytotoxicity was lower than $15 \%$ for any type of RVG29-nanoparticles, even for the highest concentration tested $(30 \mu \mathrm{M})$. Moreover, no relevant cytotoxic effects were observed for the concentration used in the permeability studies $(10 \mu \mathrm{M})$, indicating that it is safe to work in this range of concentration and, probably, may even be possible to work with higher concentrations in order to maximize the beneficial effects of quercetin in brain.

\section{BBB Permeability Study}

The hCMEC/D3 cell monolayer is a commonly used BBB model system, since it exhibits permeability values well correlated with the in vivo permeation across human BBB $(29,30)$. In this
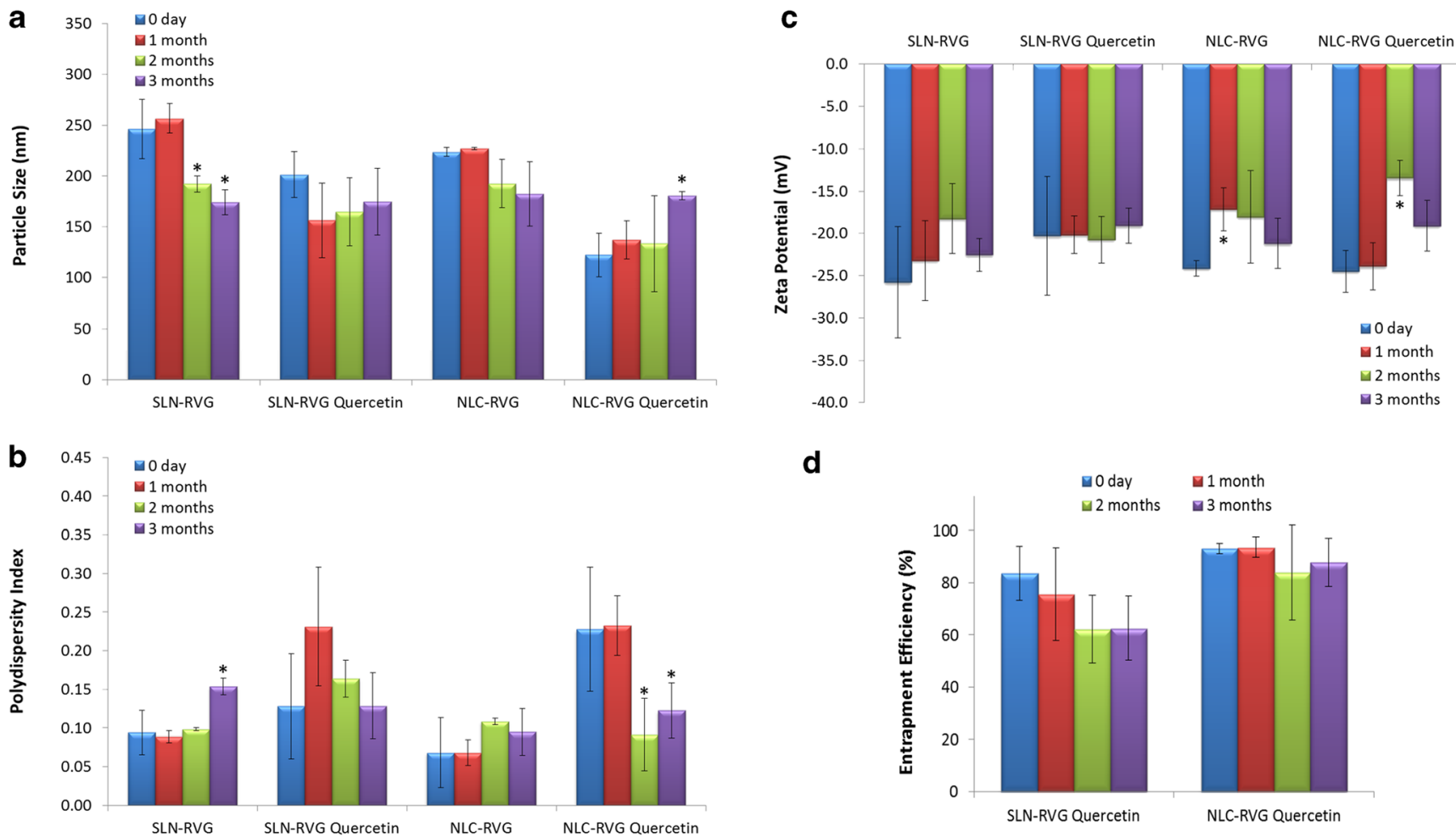

d

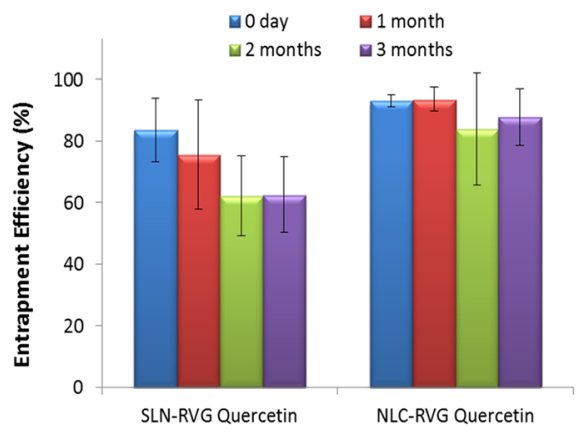

Fig. 5 Effect of time of storage on particle size (a), polydispersity index (b), zeta potential (c) and quercetin entrapment efficiency (d) of RVG29-functionalized nanoparticles. All data represent the mean \pm standard deviation $(n=3)$. * denotes statistically significant differences when compared to day $0(P<0.05)$. 

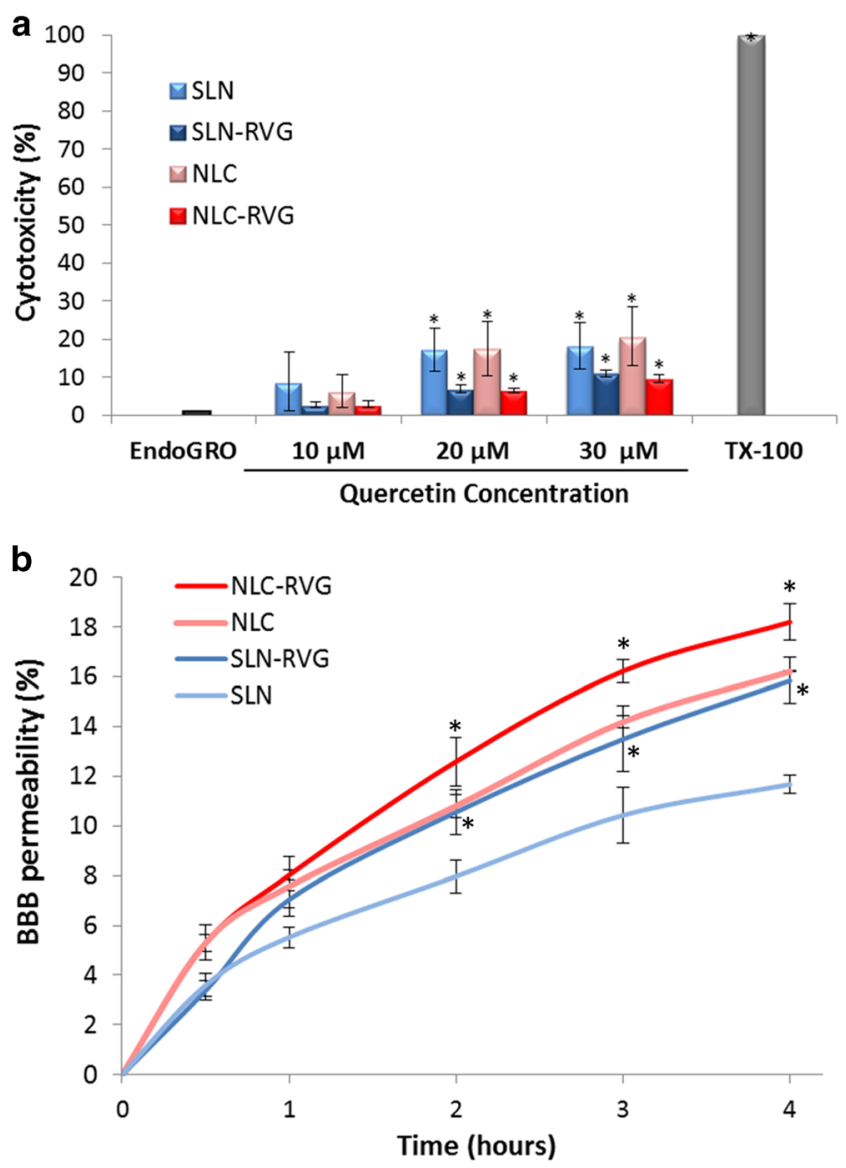

Fig. 6 (a) Cytotoxicity of nanoparticles before and after functionalization with RVG29 peptide, assessed by LDH assay on hCMEC/D3 cells. * denotes statistically significant differences $(P<0.05)$ when compared to EndoGRO medium which represents the minimum cytotoxicity. (b) Permeability study of RVG29-functionalized nanoparticles across hCMEC/D3 cell monolayer over $4 \mathrm{~h}$, mimicking BBB transport conditions. * denotes statistically significant differences $(P<0.05)$ when compared to the respective non-functionalized nanoparticles. All values represent the mean \pm standard deviation $(n=3)$.

study it was established a monolayer of hCMEC/D3 in a transwell device and the permeability rate of nonfunctionalized nanoparticles was compared to RVG29functionalized nanoparticles during $4 \mathrm{~h}$. It is necessary to note that nanoparticles were loaded with fluorescein to allow the quantification of nanoparticles in the receptor compartment. In a first glance, it was possible to observe a gradual increase of all nanoparticles content in the basolateral side of cell monolayer over time, showing that nanoparticles were progressively permeating the BBB barrier (Fig. 6b). Moreover, it was easy to conclude that NLC permeability was greater than SLN. Furthermore, a significant increase on RVG29-nanoparticles permeability (1.5fold higher) was observed when compared to non-functionalized nanoparticles, for both SLN and NLC systems. In fact, some works report that RVG29 peptide can bind to nicotinic acetylcholine receptors (nAchR) that are expressed in the BBB, enabling the crossing of this barrier (18). Besides, the expression of nAchR alpha7 on hCMEC/D3 cells has been also confirmed by real-time PCR (44). Therefore, it is possible to state that RVG29 conjugation to nanoparticles really enhances in vitro permeability through the BBB, which might lead to an increase of quercetin brain delivery in viwo.

\section{Amyloid-Beta Peptide Study}

\section{Impact of Quercetin and Nanoparticles on $A \beta(I-42)$ Fibrillation}

The quercetin ability to inhibit the fibril formation of amyloidbeta peptide is reported in the literature (10). Taking this in consideration it was evaluated if RVG29-functionalized nanoparticles loaded with quercetin could retain this ability and consequently be used in Alzheimer's disease treatment. In order to test the effect of quercetin and nanoparticles on $A \beta$ (1-42) peptide fibrillation, a ThT binding assay was performed. This compound is a fluorophore that penetrates into the cavities of the protein beta sheets and consequently can exhibit fluorescence by the space restriction rotation. Therefore, the intensity of fluorescence is proportional to the amyloid fibrils formation, so this parameter can be used to conclude about peptide aggregation $(33,35,36)$.

The interaction of quercetin and nanoparticles with the amyloid-beta peptide after $24 \mathrm{~h}$ of incubation at $37^{\circ} \mathrm{C}$ can be interpreted from Fig. 7a. It is possible to conclude that quercetin decreases the fluorescence intensity of ThT when compared to $\mathrm{A} \beta(1-42)$ peptide alone, which indicates that fibrils formation was attenuated by this compound, corroborating the study of Kim et al. (10). However, unloaded SLN and NLC seem to promote the aggregation of amyloid-beta peptide because fluorescence intensity increases, corresponding to ThT binding to the $\beta$-sheet amyloid fibrils. In fact, this result is in agreement with some previous studies reported in literature where lipid nanoparticles acted as nuclei of aggregation, promoting peptide fibrillation (45-47).

\section{Impact of Quercetin Encapsulated in Nanoparticles on $A \beta(I-42)$ Fibrillation}

Figure 7b summarizes the effect of different quercetin-loaded nanoparticles on $\mathrm{A} \beta(1-42)$ fibrillation when compared to free quercetin and unloaded nanoparticles.

It is possible to see that quercetin-loaded nanoparticles, both SLN and NLC, induce the decrease of fluorescence intensity when compared to the unloaded nanoparticles, indicating that quercetin can reverse the nucleation effect associated to the nanoparticles alone. Moreover, one can observe that all quercetin-loaded NLG and also RVG29functionalized SLN were capable of reducing the fluorescence intensity of ThT in $\mathrm{A} \beta(1-42)$, inhibiting peptide aggregation when compared to $A \beta(1-42)$ peptide alone. This evidence demonstrates that quercetin-encapsulated nanoparticles not only reverse the nucleation effect of quercetin but also inhibit 
Fig. 7 Fluorescence intensity of ThT in $A \beta(1-42)$. (a) profile over $24 \mathrm{~h}$ of incubation at $37^{\circ} \mathrm{C}$ in the absence and in the presence of free quercetin and unloaded SLN or NLC. (b) results after $24 \mathrm{~h}$ of incubation at $37^{\circ} \mathrm{C}$ with free quercetin, unloaded nanoparticles and quercetin-loaded functionalized or non-functionalized nanoparticles. SLN study in green color and NLC study in orange color. * denotes statistically significant differences $(P<0.05)$ when compared to $A \beta$ ( $1-42)$ sample alone.
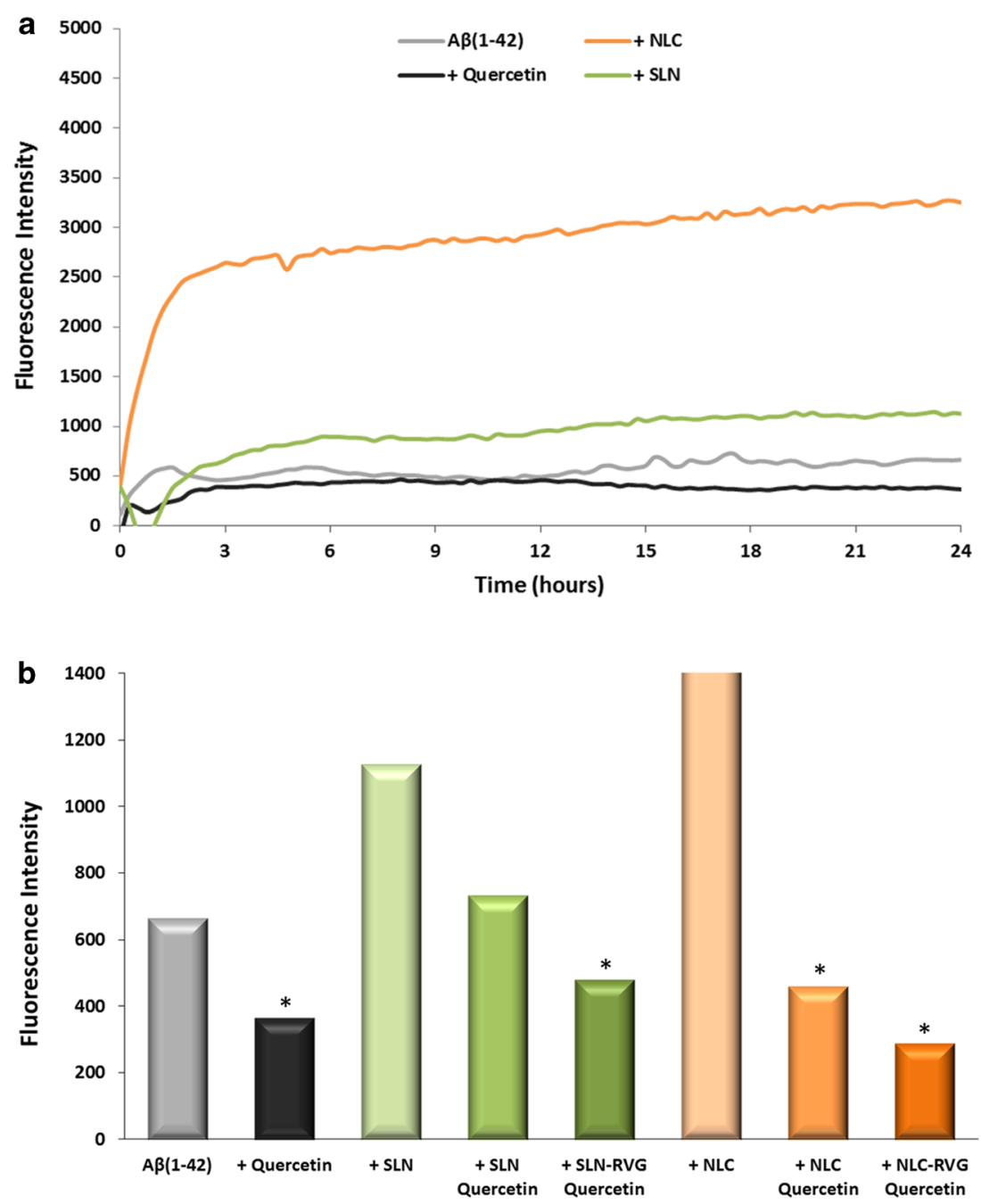

the fibril formation. It is important to refer that NLC nanoparticles showed a larger decrease in fluorescence intensity when compared to SLN nanoparticles, being therefore more efficient to inhibit fibril formation. This fact can be explained by the differences in the crystalline structure of these two types of lipid nanoparticles, which influences the capacity for accommodating and releasing quercetin from the lipid matrix. Actually, NLC have higher loading efficiency due to lattice defects that enhance quercetin entrapment, therefore showing better effects on the inhibition of fibril formation compared to SLN. Moreover, the capacity of RVG nanoparticles to inhibit peptide aggregation is also evident, clearly showing their potential to be used in a future application in the Alzheimer's disease. The mechanism that can explain the inhibition effect of quercetin and RVG29 nanoparticles towards fibrillation is probably based on the ability to block some crucial regions in the amyloid-beta peptide responsible for monomers interaction (48-51). Therefore, if these regions are blocked there will be no aggregation, being the quercetin-loaded RVG29-nanoparticles efficient in the attenuation of Alzheimer's disease by inhibiting fibrils formation.

\section{CONCLUSIONS}

This work aimed to develop nanoparticles functionalized with RVG29 peptide for quercetin delivery into the brain for the treatment of Alzheimer's disease. RVG29 is a peptide that binds specifically to the $\mathrm{nACh}$ receptors that are expressed in neuron cells and also in BBB. Quercetin is a polyphenol compound with proven antioxidant capacity and neuroprotective effects. The RVG29 functionalization of lipid nanoparticles was confirmed using NMR and FTIR spectroscopic techniques, while TEM images revealed spherical and uniform particles in the nanometer range. After functionalization, the nanosystems preserved their size below $250 \mathrm{~nm}$ and a zeta potential around $-25 \mathrm{mV}$. These properties assured their good stability over time, with no aggregation and also guaranteed the appropriate characteristics for brain delivery. The entrapment efficiency obtained was above $80 \%$, which indicated that almost all quercetin was encapsulated inside the nanoparticles. The developed nanosystems were tested in hCMEC/D3 cell line, a human BBB model commonly used due to its similarities to the real BBB. LDH assay was 
performed to study the nanoparticles cytotoxicity and biocompatibility and the results revealed that they are safe in hCMEC/D3 cells, not exceeding 15\% of cytotoxicity even for the highest concentration tested. The permeability studies performed in transwell devices with hCMEC/D3 cell monolayers showed very promising results, concerning the ability of RVG29-functionalized nanoparticles to increase in 1.5 fold the permeability across the in vitro model of $\mathrm{BBB}$ compared with non-functionalized nanoparticles. Moreover, all nanoparticles (SLN and NLC) loaded with quercetin and modified with RVG29 were capable of inhibiting the amyloid-beta aggregation, fact that was demonstrated by the significant fluorescence decrease in ThT binding assay. As a result, one can conclude that these nanosystems are able to increase the permeability across the BBB and to inhibit amyloid-beta fibrillation, demonstrating their great potential to be used as a future therapy in Alzheimer's disease.

\section{ACKNOWLEDGMENTS AND DISCLOSURES}

This work received financial support from the European Union (FEDER funds) and National Funds (FCT/MEC, Fundação para a Ciência e a Tecnologia and Ministério da Educação e Ciência) under the Partnership Agreement PT2020 UID/ QUI/50006/2013 - POCI/01/0145/FEDER/007265. Also from the UID/EQU/00511/2019-Laboratory for Process Engineering, Environment, Biotechnology and EnergyLEPABE, funded by national funds through FGT/MCTES (PIDDAC); Project POCI-01-0145-FEDER-006939, funded by FEDER funds through COMPETE2020-Programa Operacional Competitividade e Internacionalização (POCI) and by national funds (PIDDAC) through FCT/MCTES;Project "LEPABE-2-ECO-INNOVATION"-NORTE-010145-FEDER-000005, funded by Norte Portugal Regional Operational Programme (NORTE 2020), under PORTUGAL 2020 Partnership Agreement, through the European Regional Development Fund (ERDF). ARN thanks her previous Post-Doc grant under the project NORTE-010145-FEDER-000011. ARN also acknowledges ARDITI for her current Post-Doc grant (ARDITI-CQM_2017_011-PDG) under the project M1420-01-0145-FEDER-000005-CQM+ and the CQM strategic program PEst-OE/QUI/UI0674/ 2019. Andreia Granja thanks FCT for the PhD grant (SFRH/BD/130147/2017). MP thanks FCT for funding through program DL 57/2016 -Norma transitória.

The authors thank Dr. Mariana Andrade (CEMUP, UP) for technical assistance with NMR experiments and Dr. Rui Fernandes (i3S, UP) for expert help with TEM. We are also thankful to Dr. Babette Weksler from Weill Cornell Medical College (New York, USA), Dr. Ignacio A. Romero from The Open University (Milton Keynes, UK) and Dr. Pierre-Olivier Couraud from INSERM (Paris, France) for technical assistance and support with the hCMEC/D3 cell culture.
The authors declare that they have no conflict of interests.

Data Availability No raw/processed data is available.

\section{REFERENCES}

1. Heoand HJ, Lee CY. Protective effects of quercetin and vitamin C against oxidative stress-induced neurodegeneration. J Agric Food Chem. 2004;52:7514-7.

2. Zhang ZJ, Cheang LC, Wang MW, Lee SM. Quercetin exerts a neuroprotective effect through inhibition of the iNOS/NO system and pro-inflammation gene expression in PG12 cells and in zebrafish. Int J Mol Med. 2011;27:195-203.

3. Dok-Go H, Lee KH, Kim HJ, Lee EH, Lee J, Song YS, et al. Neuroprotective effects of antioxidative flavonoids, quercetin, $(+)$ dihydroquercetin and quercetin 3-methyl ether, isolated from Opuntia ficus-indica var. saboten. Brain Res. 2003;965:130-6.

4. Sagara Y, Vanhnasy J, Maher P. Induction of PC12 cell differentiation by flavonoids is dependent upon extracellular signal-regulated kinase activation. J Neurochem. 2004;90:1 144-55.

5. Min YD, Choi CH, Bark H, Son HY, Park HH, Lee S, et al. Quercetin inhibits expression of inflammatory cytokines through attenuation of NF-kappaB and p38 MAPK in HMC-1 human mast cell line. Inflamm Res: Official Journal of the European Histamine Research Society [et al]. 2007;56:210-5.

6. Kimata M, Shichijo M, Miura T, Serizawa I, Inagaki N, Nagai H. Effects of luteolin, quercetin and baicalein on immunoglobulin Emediated mediator release from human cultured mast cells. Clin Exp Allergy: J Br Soc Allergy Clin Immunol. 2000;30:501-8.

7. Afanas'ev IB, Dorozhko AI, Brodskii AV, Kostyuk VA, Potapovitch AI. Chelating and free radical scavenging mechanisms of inhibitory action of rutin and quercetin in lipid peroxidation. Biochem Pharmacol. 1989;38:1763-9.

8. van Acker SA, van Balen GP, van den Berg DJ, Bast A, van der Vijgh WJ. Influence of iron chelation on the antioxidant activity of flavonoids. Biochem Pharmacol. 1998;56:935-43.

9. Ansari MA, Abdul HM, Joshi G, Opii WO, Butterfield DA. Protective effect of quercetin in primary neurons against Abeta(142): relevance to Alzheimer's disease. J Nutr Biochem. 2009;20: 269-75.

10. Kim H, Park BS, Lee KG, Choi CY, Jang SS, Kim YH, et al. Effects of naturally occurring compounds on fibril formation and oxidative stress of beta-amyloid. J Agric Food Chem. 2005;53: 8537-41.

11. Maria S-GA, Ignacio M-MJ, Ramírez-Pineda Jose R, Marisol L-R, Edison O, Patricia G-GG. The flavonoid quercetin ameliorates Alzheimer's disease pathology and protects cognitive and emotional function in aged triple transgenic Alzheimer's disease model mice. Neuropharmacology. 2015;93:134-45.

12. Guoand Y, Bruno RS. Endogenous and exogenous mediators of quercetin bioavailability. J Nutr Biochem. 2015;26:201-10.

13. Almeida AF, Borge GIA, Piskula M, Tudose A, Tudoreanu L, Valentova K, et al. Bioavailability of quercetin in humans with a focus on interindividual variation. Compr Rev Food Sci Food Saf. 2018;17:714-31.

14. Soni S, Ruhela RK, Medhi B. Nanomedicine in central nervous system (CNS) disorders: a present and future prospective. Adv Pharm Bull. 2016;6:319-35.

15. Chowdhury A, Kunjiappan S, Panneerselvam T, Somasundaram B, Bhattacharjee C. Nanotechnology and nanocarrier-based approaches on treatment of degenerative diseases. Int Nano Lett. 2017;7:91-122. 
16. Le Novere N, Corringer PJ, Changeux JP. The diversity of subunit composition in nAChRs: evolutionary origins, physiologic and pharmacologic consequences. J Neurobiol. 2002;53:447-56.

17. Gotti C, Clementi F, Fornari A, Gaimarri A, Guiducci S, Manfredi $\mathrm{I}$, et al. Structural and functional diversity of native brain neuronal nicotinic receptors. Biochem Pharmacol. 2009;78:703-11.

18. Hawkins BT, Egleton RD, Davis TP. Modulation of cerebral microvascular permeability by endothelial nicotinic acetylcholine receptors. Am J Physiol Heart Circ Physiol. 2005;289:H212-9.

19. Lafon M. Rabies virus receptors. J Neurovirol. 2005;1 1:82-7.

20. Kumar P, Wu H, McBride JL, Jung KE, Kim MH, Davidson BL, et al. Transvascular delivery of small interfering RNA to the central nervous system. Nature. 2007;448:39-43.

21. Liu Y, Huang R, Han L, Ke W, Shao K, Ye L, et al. Braintargeting gene delivery and cellular internalization mechanisms for modified rabies virus glycoprotein RVG29 nanoparticles. Biomaterials. 2009;30:4195-202.

22. Hua HC, Zhang XM, Mu HJ, Meng QQ Jiang Y, Wang YY, et al. RVG29-modified docetaxel-loaded nanoparticles for braintargeted glioma therapy. Int J Pharm. 2018;543:179-89.

23. You LH, Wang J, Liu TQ, Zhang YL, Han XX, Wang T, et al. Targeted brain delivery of rabies virus glycoprotein 29-modified deferoxamine-loaded nanoparticles reverses functional deficits in Parkinsonian mice. ACS Nano. 2018;12:4123-39.

24. Oswald M, Geissler S, Goepferich A. Targeting the central nervous system (CNS): a review of rabies virus-targeting strategies. Mol Pharm. 2017;14:2177-96.

25. Neves AR, Lucio M, Martins S, Lima JL, Reis S. Novel resveratrol nanodelivery systems based on lipid nanoparticles to enhance its oral bioavailability. Int J Nanomedicine. 2013;8:177-87.

26. Neves AR, Queiroz JF, Reis S. Brain-targeted delivery of resveratrol using solid lipid nanoparticles functionalized with apolipoprotein E. J Nanobiotechnol. 2016;14:27.

27. Neves AR, QueirozJF, Weksler B, Romero IA, Couraud PO, Reis S. Solid lipid nanoparticles as a vehicle for brain-targeted drug delivery: two new strategies of functionalization with apolipoprotein E. Nanotechnology. 2015;26:495103

28. Neves AR, QueirozJF, Lima SAC, Reis S. Apo E-functionalization of solid lipid nanoparticles enhances brain drug delivery: uptake mechanism and transport pathways. Bioconjug Chem. 2017;28: 995-1004.

29. Weksler B, Romero IA, Couraud PO. The hCMEC/D3 cell line as a model of the human blood brain barrier. Fluids Barriers CNS. 2013;10:16.

30. Weksler BB, Subileau EA, Perriere N, Charneau P, Holloway K, Leveque M, et al. Blood-brain barrier-specific properties of a human adult brain endothelial cell line. FASEB J: Official Publication FASEB. 2005; 19:1872-4.

31. Poller B, Gutmann H, Krahenbuhl S, Weksler B, Romero I, Couraud PO, et al. The human brain endothelial cell line hCMEC/D3 as a human blood-brain barrier model for drug transport studies. J Neurochem. 2008;107:1358-68.

32. Sabateand R, Estelrich J. Stimulatory and inhibitory effects of alkyl bromide surfactants on beta-amyloid fibrillogenesis. Langmuir. 2005;21:6944-9.

33. Nilsson MR. Techniques to study amyloid fibril formation in vitro. Methods. 2004;34:151-60.

34. Rocha S, Cardoso I, Borner H, Pereira MC, Saraiva MJ, Coelho M. Design and biological activity of beta-sheet breaker peptide conjugates. Biochem Bioph Res Commun. 2009;380:397-401.

35. Jameson LP, Smith NW, Dzyuba SV. Dye-binding assays for evaluation of the effects of small molecule inhibitors on amyloid (abeta) self-assembly. ACS Chem Neurosci. 2012;3:807-19.
36. LeVine H 3rd. Thioflavine T interaction with synthetic Alzheimer's disease beta-amyloid peptides: detection of amyloid aggregation in solution. Protein Sci. 1993;2:404-10.

37. Yan Z, Yang Y, Wei X, Zhong J, Wei D, Liu L, et al. Tumorpenetrating peptide mediation: an effective strategy for improving the transport of liposomes in tumor tissue. Mol Pharm. 2014;11: 218-25.

38. Wei X, Zhan C, Chen X, Hou J, Xie C, Lu W. Retro-inverso isomer of Angiopep-2: a stable d-peptide ligand inspires braintargeted drug delivery. Mol Pharm. 2014;11:3261-8.

39. Gaumet M, Vargas A, Gurny R, Delie F. Nanoparticles for drug delivery: the need for precision in reporting particle size parameters. Eur J Pharm Biopharm: Official J Arbeitsgemeinschaft fur Pharmazeutische Verfahrenstechnik eV. 2008;69:1-9.

40. Naseri N, Valizadeh H, Zakeri-Milani P. Solid lipid nanoparticles and nanostructured lipid carriers: structure. Prep Appl Adv Pharm Bull. 2015;5:305-13.

41. Freitasand C, Muller RH. Correlation between long-term stability of solid lipid nanoparticles (SLN) and crystallinity of the lipid phase. Eur J Pharm Biopharm: Official J Arbeitsgemeinschaft fur Pharmazeutische Verfahrenstechnik eV. 1999;47:125-32.

42. Burdand JF, Usategui-Gomez M. A colorimetric assay for serum lactate dehydrogenase. Clin Chim Acta; Int J Clin Chem. 1973;46: 223-7.

43. Korzeniewskiand C, Callewaert DM. An enzyme-release assay for natural cytotoxicity. J Immunol Methods. 1983;64:313-20.

44. Phoolcharoen W, Prehaud C, van Dolleweerd CJ, Both L, da Costa A, Lafon M, et al. Enhanced transport of plant-produced rabies single-chain antibody-RVG peptide fusion protein across an in cellulo blood-brain barrier device. Plant Biotechnol J. 2017;15: 1331-9.

45. Loureiro JA, Rocha S, Pereira Mdo C. Charged surfactants induce a non-fibrillar aggregation pathway of amyloid-beta peptide. J Pept Sci: Official Publication Eur Pept Soc. 2013;19:581-7.

46. Loureiro JA, Gomes B, Fricker G, Cardoso I, Ribeiro CA, Gaiteiro C, et al. Dual ligand immunoliposomes for drug delivery to the brain. Colloid Surface B, Biointerfaces. 2015;134:213-9.

47. Loureiro JA, Andrade S, Duarte A, Neves AR, Queiroz JF, Nunes C, et al. Resveratrol and grape extract-loaded solid lipid nanoparticles for the treatment of Alzheimer's disease. Molecules (Basel, Switzerland). 2017;22:277.

48. Petkova AT, Ishii Y, Balbach JJ, Antzutkin ON, Leapman RD, Delaglio F, et al. A structural model for Alzheimer's beta amyloid fibrils based on experimental constraints from solid state NMR. Proc Natl Acad Sci U S A. 2002;99:16742-7.

49. Pike CJ, Walencewicz-Wasserman AJ, Kosmoski J, Cribbs DH, Glabe CG, Cotman CW. Structure-activity analyses of betaamyloid peptides: contributions of the beta 25-35 region to aggregation and neurotoxicity. J Neurochem. 1995;64:253-65.

50. Torok M, Milton S, Kayed R, Wu P, McIntire T, Glabe GG, et al. Structural and dynamic features of Alzheimer's Abeta peptide in amyloid fibrils studied by site-directed spin labeling. J Biol Chem. 2002;277:40810-5.

51. Antzutkin ON, Balbach JJ, Tycko R. Site-specific identification of non-beta-strand conformations in Alzheimer's beta-amyloid fibrils by solid-state NMR. Biophys J. 2003;84:3326-35.

Publisher's Note Springer Nature remains neutral with regard to jurisdictional claims in published maps and institutional affiliations. 